\title{
A Metallocene-Pyrrolidinopyridine Nucleophilic Catalyst for Asymmetric Synthesis
}

\author{
Huy V. Nguyen, David C. D. Butler and Christopher J. Richards*
}

School of Biological and Chemical Sciences, Queen Mary, University of London, Mile End Road, E1 4NS,

$U K$.

\section{Supporting Information}

\section{Index}

\section{Page}

Synthesis of 4.

Synthesis of 7.

Synthesis of 1.

General method of alcohol acetylation.

Synthesis of $\mathbf{1 0 b}$.

General method of asymmetric Steglich rearrangement.

Synthesis of 12a.

ORTEP representation of 4 .

${ }^{1} \mathrm{H}$ NMR spectrum of 4 .

${ }^{1} \mathrm{H}$ NMR spectrum of 7 .

${ }^{1} \mathrm{H}$ NMR spectrum of $\mathbf{1}$.

${ }^{1} \mathrm{H}$ NMR spectrum of $\mathbf{1 0 b}$.

${ }^{1} \mathrm{H}$ NMR spectrum of $\mathbf{1 1 b}$.

${ }^{1} \mathrm{H}$ NMR spectrum of $\mathbf{1 2 a}$.

HPLC analysis of $\mathbf{1 1 b .}$

HPLC analysis of 12a. 
General considerations. Petroleum ether refers to that fraction boiling in the range $40-60{ }^{\circ} \mathrm{C}$. THF was distilled from sodium benzophenone ketyl, and toluene from sodium wire. Silica gel 40-63 $\mu \mathrm{m}$ was used for chromatography. All reactions were performed under nitrogen. Butyllithium was used as a $2.5 \mathrm{M}$ solution in hexanes, DIBAL as a $1.0 \mathrm{M}$ solution in hexanes and $\mathrm{ZnCl}_{2}$ as a $0.5 \mathrm{M}$ solution in THF.

Synthesis of 4. To a solution of $\left(\eta^{5}\right.$-iodocyclopentadienyl $)\left(\eta^{4}-\right.$ tetraphenylcyclobutadiene)cobalt(I) $)^{1}(0.250 \mathrm{~g}, 0.41 \mathrm{mmol})$ in dry toluene $(4 \mathrm{~mL})$ at $c a .-80$ ${ }^{\circ} \mathrm{C}$ was added $\mathrm{BuLi}(0.18 \mathrm{~mL}, 0.45 \mathrm{mmol})$ and the resulting solution warmed to room temperature. After stirring for $20 \mathrm{~min}$, a solution of $\mathrm{ZnCl}_{2}(0.067 \mathrm{~g}, 0.49 \mathrm{mmol})$ in THF (2 $\mathrm{mL}$ ) was added via cannular, and after stirring for a further $10 \mathrm{~min}$, 3-bromo-4pyrrolidinopyridine $^{2}(0.094 \mathrm{~g}, 0.41 \mathrm{mmol})$ was added to the resultant orange solution. In a separate flask a suspension of $\mathrm{PdCl}_{2}\left(\mathrm{PPh}_{3}\right)_{2}(0.060 \mathrm{~g}, 0.09 \mathrm{mmol})$ in toluene $(2 \mathrm{~mL})$ at room temperature was treated with $\operatorname{DIBAL}(0.17 \mathrm{~mL}, 0.17 \mathrm{mmol})$ and stirring continued for a further 20 minutes. The resulting black solution was added via cannular to the other reaction vessel and the resultant mixture was heated at $100{ }^{\circ} \mathrm{C}$ for 18 hours. After cooling and removal of the solvent in vacuo, the resulting brown oil was column chromatograhed (EtOAc to 1.5\% $\left.\mathrm{NEt}_{3} / \mathrm{EtOAc}\right)$ to give $4(0.065 \mathrm{~g}, 25 \%)$ as an orange crystalline solid: Mp 117-120 ${ }^{\circ} \mathrm{C}$. IR $\left(\mathrm{CH}_{2} \mathrm{Cl}_{2}\right) v_{\max } 1639,1585 \mathrm{~cm}^{-1} .{ }^{1} \mathrm{H}$ NMR $\left(270 \mathrm{MHz}, \mathrm{CDCl}_{3}\right) 1.70-1.77(4 \mathrm{H}, \mathrm{m}), 2.95-3.02$ (4 H, m), $4.62(2 \mathrm{H}, \mathrm{t}, J$ 2), 4.88 (2 H, t, J 2), $6.44(1 \mathrm{H}, \mathrm{d}, J 6), 7.10-7.27(12 \mathrm{H}, \mathrm{m}), 7.32$ $7.40(8 \mathrm{H}, \mathrm{m}), 7.84(1 \mathrm{H}, \mathrm{s}), 7.97(1 \mathrm{H}, \mathrm{d}, J 6) .{ }^{13} \mathrm{C}\left\{{ }^{1} \mathrm{H}\right\} \mathrm{NMR}\left(68 \mathrm{MHz}, \mathrm{CDCl}_{3}\right)$ 25.4, 50.5, $75.1,83.5,83.7,99.3,108.4,117.0,126.3,127.6,128.0,128.8,135.9,147.1,152.6,154.4$. High-resolution MS (m/z, FAB): found for $\mathrm{MH}^{+}$627.2228. $\mathrm{C}_{42} \mathrm{H}_{36} \mathrm{CoN}_{2}$ requires 627.2210. 
Synthesis of 7. To a solution of 4-amino-3-bromopyridine ( $0.870 \mathrm{~g}, 5.03 \mathrm{mmol})$ in THF (30 $\mathrm{mL})$ was added $\mathrm{NaH}(60 \%$ dispersion in mineral oil - $0.502 \mathrm{~g}, 12.6 \mathrm{mmol})$ and the resulting solution stirred at room temperature for 3 hours. Following the addition of a solution of $(S, S)$ 2,5-bis(methylsulfonyloxy)hexane $(0.689 \mathrm{~g}, 2.51 \mathrm{mmol}$ - synthesised in $91 \%$ yield using the procedure previously reported $\left.{ }^{3}\right)$ in THF $(25 \mathrm{~mL})$, the resulting reaction mixture was heated at reflux for 20 hours. After cooling, quenching with water $(10 \mathrm{~mL})$ and extraction with $\mathrm{CH}_{2} \mathrm{Cl}_{2}$ $(3 \times 30 \mathrm{~mL})$, the combined organic extracts were dried $\left(\mathrm{MgSO}_{4}\right)$, filtered and evaporated in vacuo. Column chromatography $\left(0.25 \% \mathrm{NEt}_{3} / 5 \% \mathrm{MeOH} / \mathrm{CH}_{2} \mathrm{Cl}_{2}\right)$ gave $7(0.307 \mathrm{~g}, 48 \%$ yield) as a yellow oil that was further purified by Kugelrohr distillation to give a pale yellow oil: $[\alpha]_{\mathrm{D}}^{22}=+135(\mathrm{MeOH}, c 1.0)$. IR (film) $v_{\max } 2910,1575,1480 \mathrm{~cm}^{-1} .{ }^{1} \mathrm{H}$ NMR $(270 \mathrm{MHz}$, $\left.\mathrm{CDCl}_{3}\right) 0.97(6 \mathrm{H}, \mathrm{d}, J$ 6), $1.53-1.65(2 \mathrm{H}, \mathrm{m}), 2.16-2.22(2 \mathrm{H}, \mathrm{m}), 4.33-4.46(2 \mathrm{H}, \mathrm{m})$, $6.64(1 \mathrm{H}, \mathrm{d}, J 5), 8.15(1 \mathrm{H}, \mathrm{d}, J 5), 8.41(1 \mathrm{H}, \mathrm{s}) .{ }^{13} \mathrm{C}\left\{{ }^{1} \mathrm{H}\right\} \mathrm{NMR}\left(68 \mathrm{MHz}, \mathrm{CDCl}_{3}\right)$ 19.8, 20.7, 30.1, 32.4, 47.8, 55.0, 106.1, 111.9, 147.6, 151.7, 153.4. High-resolution MS ( $\mathrm{m} / \mathrm{z}$, ES): found for $\mathrm{MH}^{+}$255.0492. $\mathrm{C}_{11} \mathrm{H}_{16}{ }^{79} \mathrm{BrN}_{2}$ requires 255.0491 .

Synthesis of 1. To a solution of $\left(\eta^{5}\right.$-iodocyclopentadienyl $)\left(\eta^{4}-\right.$ tetraphenylcyclobutadiene)cobalt(I) $(0.250 \mathrm{~g}, 0.41 \mathrm{mmol})$ in toluene $(4 \mathrm{~mL})$ at $c a .-80{ }^{\circ} \mathrm{C}$ was added $\mathrm{BuLi}(0.18 \mathrm{~mL}, 0.45 \mathrm{mmol})$ and the resulting solution warmed to room temperature. After stirring for $30 \mathrm{~min}$, the reaction was recooled to $c a .-80{ }^{\circ} \mathrm{C}$ and a solution of $\mathrm{ZnCl}_{2}$ in THF (0.95 mL, $0.48 \mathrm{mmol})$ was added via syringe. After warming to room temperature, addition of $7(0.125 \mathrm{~g}, 0.49 \mathrm{mmol})$ was followed by addition of the catalyst prepared in a separate flask by suspending $\mathrm{PdCl}_{2}\left(\mathrm{PPh}_{3}\right)_{2}(0.014 \mathrm{~g}, 0.02 \mathrm{mmol})$ in toluene $(4 \mathrm{~mL})$ at room temperature, followed by addition of DIBAL $(0.04 \mathrm{~mL}, 0.04 \mathrm{mmol})$ and stirring for a further 20 minutes. The resultant mixture was heated at $100{ }^{\circ} \mathrm{C}$ for $22 \mathrm{~h}$, cooled, quenched with EtOAc $(10 \mathrm{~mL})$ and filtered through a short plug of $\mathrm{SiO}_{2}$ prior to removal of the solvent in 
vacuo. Column chromatography $\left(0.25 \% \mathrm{NEt}_{3} / 5 \% \mathrm{MeOH} / \mathrm{CH}_{2} \mathrm{Cl}_{2}\right)$ gave $\mathbf{1}(0.134 \mathrm{~g}, 50 \%)$ as an orange crystalline solid: Mp $120-125^{\circ} \mathrm{C} .[\alpha]_{\mathrm{D}}^{22}=-340\left(\mathrm{MeOH}, c\right.$ 0.05). IR (nujol) $v_{\max } 3100$, $1500 \mathrm{~cm}^{-1} .{ }^{1} \mathrm{H}$ NMR $\left(270 \mathrm{MHz}, \mathrm{CDCl}_{3}\right) 0.46$ (3 H, br), $0.97(3 \mathrm{H}, \mathrm{br}), 1.93$ - $2.06(4 \mathrm{H}, \mathrm{m})$, 3.67 - $3.78(2 \mathrm{H}, \mathrm{m}), 4.58(1 \mathrm{H}, \mathrm{s}), 4.73(1 \mathrm{H}, \mathrm{s}), 4.92(1 \mathrm{H}, \mathrm{s}), 5.14(1 \mathrm{H}, \mathrm{s}), 6.61(1 \mathrm{H}, \mathrm{d}, J 5)$, $7.08-7.38(20 \mathrm{H}, \mathrm{m}), 7.76(1 \mathrm{H}, \mathrm{s}), 8.02(1 \mathrm{H}, \mathrm{d}, J 5) .{ }^{13} \mathrm{C}\left\{{ }^{1} \mathrm{H}\right\}$ NMR $\left(100 \mathrm{MHz}, \mathrm{CDCl}_{3}-\right.$ pyrrolidine methyl and methylenes not observed) $74.4,81.5,82.1,82.2,82.9,95.4,113.8$, 122.0, 125.2, 126.9, 127.7, 134.8, 145.4, 150.2, 151.3. High-resolution MS ( $m / z$, ES): found for $\mathrm{MH}^{+}$655.2521. $\mathrm{C}_{44} \mathrm{H}_{40} \mathrm{CoN}_{2}$ requires 655.2518.

General method of alcohol acetylation. 1-(1-Naphthyl)ethanol 8 (0.086 g, $0.5 \mathrm{mmol})$ was dissolved in $\mathrm{CH}_{2} \mathrm{Cl}_{2}(2 \mathrm{~mL})$ and the reaction vessel cooled to $-78^{\circ} \mathrm{C}$. To this was added the catalyst $(0.005 \mathrm{mmol})$ dissolved in toluene $(1 \mathrm{~mL})$ followed by triethylamine $(0.07 \mathrm{~mL}, 0.5$ mmol) and acetic anhydride $(0.12 \mathrm{~mL}, 1.3 \mathrm{mmol})$. The reaction mixture was stirred and maintained at $-78{ }^{\circ} \mathrm{C}$ for 5 hours. After quenching with methanol, the solvent was removed in vacuo and ${ }^{1} \mathrm{H}$ NMR was used to determine the level of conversion $\left(\mathbf{8}: \mathrm{CH}\left(\mathrm{CH}_{3}\right) \mathrm{OH}=5.63\right.$ ppm, 9: $\left.\mathrm{CH}\left(\mathrm{CH}_{3}\right) \mathrm{OAc}=6.65 \mathrm{ppm}\right)$. The product 9 was isolated by column chromatography (5\% ethyl acetate /petroleum ether to $25 \%$ ethyl acetate/petroleum ether to $2 \%$ $\mathrm{MeOH} / \mathrm{CH}_{2} \mathrm{Cl}_{2}$ ), and the enantiomeric excess determined by gas chromatography (ChirasilDex CB $25 \mathrm{~m} \times 0.25 \mathrm{~mm}$ ), starting temperature $130{ }^{\circ} \mathrm{C}$, initial time $1 \mathrm{~min}$, rate $1{ }^{\circ} \mathrm{C} / \mathrm{min}$, final temperature $180^{\circ} \mathrm{C}$, final time 10 minutes. Retention time $45.76 \mathrm{~min}(S-8)$ and $47.74 \mathrm{~min}(R$ 8).

Synthesis of 10b. 2-(4-Methoxyphenyl)-4-methyloxazalone ${ }^{4}(1.00 \mathrm{~g}, 4.9 \mathrm{mmol})$ was dissolved in THF $(20 \mathrm{~mL})$ and cooled to $0{ }^{\circ} \mathrm{C}$. To this was added $\mathrm{Et}_{3} \mathrm{~N}(0.75 \mathrm{~mL}, 5.4 \mathrm{mmol})$ followed by 2,2,2-trichloro-1,1-dimethylethyl chloroformate (1.24 g, $5.2 \mathrm{mmol})$. The reaction 
mixture was allowed to stir for $30 \mathrm{~min}$ at $0{ }^{\circ} \mathrm{C}$ and then poured into water and extracted with diethyl ether $(25 \mathrm{~mL})$. The combined organic layers were washed with $0.1 \mathrm{M} \mathrm{HCl}$, sat aqueous $\mathrm{NaHCO}_{3}$ and brine (25 mL of each), dried with $\mathrm{MgSO}_{4}$ and the solvent removed in vacuo. Column chromatography (1:1 diethyl ether/petroleum ether) gave $\mathbf{1 0 b}(1.29 \mathrm{~g}, 65 \%$ yield $)$ as a colourless powder: Mp $97-101{ }^{\circ} \mathrm{C}$. IR (nujol) $v_{\max }$ 1782, 1674, $1614 \mathrm{~cm}^{-1} .{ }^{1} \mathrm{H}$ NMR (270 $\left.\mathrm{MHz}, \mathrm{CDCl}_{3}\right) 2.00(6 \mathrm{H}, \mathrm{s}), 2.12(3 \mathrm{H}, \mathrm{s}), 3.85(3 \mathrm{H}, \mathrm{s}), 6.92(2 \mathrm{H}, \mathrm{d}, J$ 8.6), $7.86(2 \mathrm{H}, \mathrm{d}, J$ 8.6). ${ }^{13} \mathrm{C}\left\{{ }^{1} \mathrm{H}\right\}$ NMR (68 MHz, $\left.\mathrm{CDCl}_{3}\right)$ 10.4, 21.1, 55.4, 92.3, 104.6, 114.2, 119.9, 120.2, 127.6, 145.4, 148.5, 155.1, 161.4. High-resolution MS (m/z, ES) $\mathrm{MH}^{+} 408.0172$. $\mathrm{C}_{16} \mathrm{H}_{17}{ }^{35} \mathrm{Cl}_{3} \mathrm{NO}_{5}$ requires 408.0167 .

General method of asymmetric Steglich rearrangement. A stirred solution of substrates $10 \mathrm{a}$ or $10 \mathrm{~b}(0.5 \mathrm{mmol})$ in the relevant solvent $(5 \mathrm{~mL})$ was cooled to $0{ }^{\circ} \mathrm{C}$ and to this was added a solution of the catalyst $1(0.0033 \mathrm{~g}, 0.005 \mathrm{mmol})$ in the same solvent $(2 \mathrm{~mL})$, and the reaction mixture made up to $0.05 \mathrm{M}$ by addition of a further $3 \mathrm{~mL}$ of solvent. The reaction was then stirred at $0{ }^{\circ} \mathrm{C}$ and progress monitored by TLC (1:1 diethyl ether/petroleum ether). On completion, the catalyst was removed by filtering the reaction mixture through a $2 \mathrm{~cm}$ silica plug washing with $\mathrm{CH}_{2} \mathrm{Cl}_{2}$ followed by ethyl acetate. The solvent was removed in vacuo and subsequent column chromatography (1:1 diethyl ether/petroleum ether) gave either $\mathbf{1 1 a}^{4}$ or 11b as colourless oils. 11a: $[\alpha]_{\mathrm{D}}{ }^{25}=+40\left(\mathrm{CHCl}_{3}, c 0.95\right)\left(\right.$ Lit. $^{4}{ }^{4}[\alpha]_{\mathrm{D}}{ }^{20}=-55\left(\mathrm{CHCl}_{3}, c 0.95\right)$ for a sample of $(S)$-11a of $91 \%$ e.e.). 11b (0.13 g, $64 \%$ yield). HPLC (Chiralcel OD, $4.6 \mathrm{~mm}$ $\times 25 \mathrm{~m}, 90: 10$ hexane/isopropanol, $1 \mathrm{~mL} / \mathrm{min}$ ) $\mathrm{T}_{\mathrm{R}}=13.25 \mathrm{~min}$ (major), $\mathrm{T}_{\mathrm{R}}=15.56 \mathrm{~min}$ (minor), $76 \%$ e.e. $[\alpha]_{\mathrm{D}}^{22}=+18\left(\mathrm{CHCl}_{3}, c 1.0\right)$. IR (film) $v_{\max } 1825,1762,1651,1608 \mathrm{~cm}^{-1} .{ }^{1} \mathrm{H}$ NMR (270 MHz, CDCl $) 1.73(3 \mathrm{H}, \mathrm{s}), 1.87(3 \mathrm{H}, \mathrm{s}), 1.90(3 \mathrm{H}, \mathrm{s}), 3.87(3 \mathrm{H}, \mathrm{s}), 6.96(2 \mathrm{H}, \mathrm{d}, J$ 8.7), 7.94 (2 H, d, J 8.7). ${ }^{13} \mathrm{C}\left\{{ }^{1} \mathrm{H}\right\}$ NMR (68 MHz, $\left.\mathrm{CDCl}_{3}\right)$ 19.4, 21.0, 21.2, 55.6, 73.6, 90.8, $105.8,114.4,117.4,125.5,129,130.3,163.8,174.9$. 
Synthesis of 12a. To a solution of $11 \mathrm{a}(0.100 \mathrm{~g}, 0.29 \mathrm{mmol})$ in $\mathrm{CH}_{2} \mathrm{Cl}_{2}(10 \mathrm{~mL})$ was added $\mathrm{MeOH}(2 \mathrm{~mL}), \mathrm{NEt}_{3}(0.03 \mathrm{~g}, 0.3 \mathrm{mmol})$ and $\mathrm{SiO}_{2}(1.5 \mathrm{~g})$. The resulting mixture was stirred for $30 \mathrm{~min}$, the solvent removed in vacuo, and the residual silica added to the top of a chromatography column followed by subsequent elution $\left(\mathrm{CH}_{2} \mathrm{Cl}_{2}\right.$ to $\left.2 \% \mathrm{MeOH} / \mathrm{CH}_{2} \mathrm{Cl}_{2}\right)$ to give product 12a $(0.060 \mathrm{~g}, 55 \%$ yield) as a colourless oil. HPLC (Chiralcel OD, $4.6 \mathrm{~mm} \times$ $25 \mathrm{~m}, 90: 10$ hexane/isopropanol, $1 \mathrm{~mL} / \mathrm{min}$ ) $\mathrm{T}_{\mathrm{R}}=28.02 \mathrm{~min}\left(\right.$ minor), $\mathrm{T}_{\mathrm{R}}=30.48 \mathrm{~min}$ (major), $72 \%$ e.e. $[\alpha]_{\mathrm{D}}^{22}=-16.7\left(\mathrm{CHCl}_{3}, c 0.8\right)$. IR (film) $v_{\max } 3400,1739,1670 \mathrm{~cm}^{-1} .{ }^{1} \mathrm{H}$ NMR $(270$ $\left.\mathrm{MHz}, \mathrm{CDCl}_{3}\right) 1.86(3 \mathrm{H}, \mathrm{s}), 3.71(3 \mathrm{H}, \mathrm{s}), 3.84(3 \mathrm{H}, \mathrm{s}), 5.24(2 \mathrm{H}, \mathrm{s}), 6.92(2 \mathrm{H}, \mathrm{d}, J$ 8.4), 7.28 - $7.32(5 \mathrm{H}, \mathrm{m}), 7.43(1 \mathrm{H}, \mathrm{brs}), 7.76\left(2 \mathrm{H}, \mathrm{d}, J\right.$ 8.4). ${ }^{13} \mathrm{C}\left\{{ }^{1} \mathrm{H}\right\} \mathrm{NMR}\left(68 \mathrm{MHz}, \mathrm{CDCl}_{3}\right)$ 21.17, $53.50,55.52,63.25,68.10,113.9,125.7,128.1,128.5,128.6,129.1,135.1,162.7,165.6$, 168.8. High-resolution MS (m/z, ES) $\mathrm{MH}^{+} 372.1439 . \mathrm{C}_{20} \mathrm{H}_{22} \mathrm{NO}_{6}$ requires 372.1442 .

\section{References.}

1. Rausch, M. D.; Genetti, R. A. J. Org. Chem. 1970, 35, 3888.

2. Spivey, A. C.; Fekner, T.; Spey, S. E.; Adams, H. J. Org. Chem. 1999, 64, 9430.

3. Zwaagstra, M. E.; Meetsma, A.; Feringa, B. L. Tetrahedron: Asymmetry 1993, 4, 2163.

4. Ruble, J. C.; Fu, G. C. J. Am. Chem. Soc. 1998, 120, 11532. 
ORTEP representation of 4 .

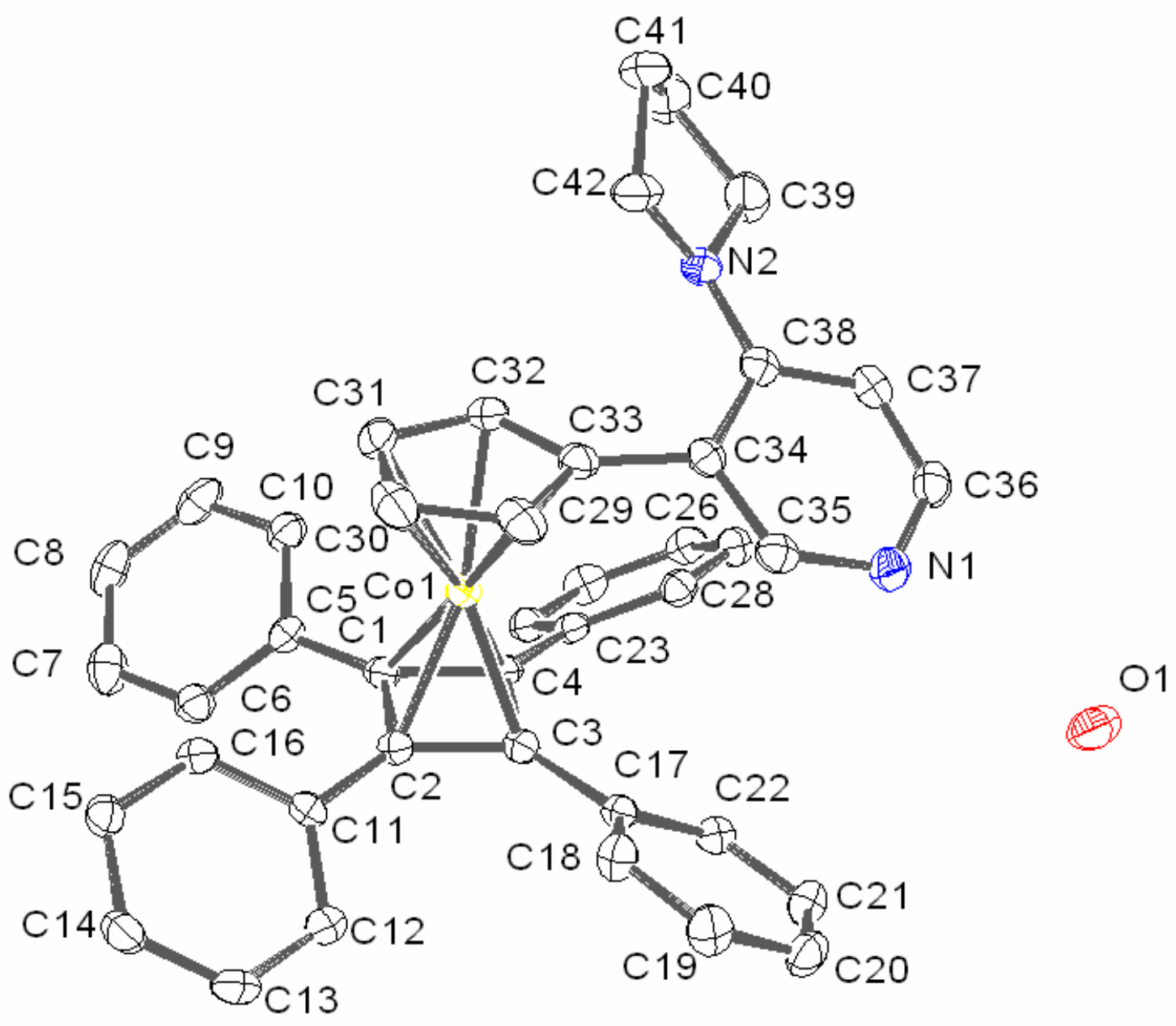


${ }^{1} \mathrm{H}$ NMR spectrum of 4 .
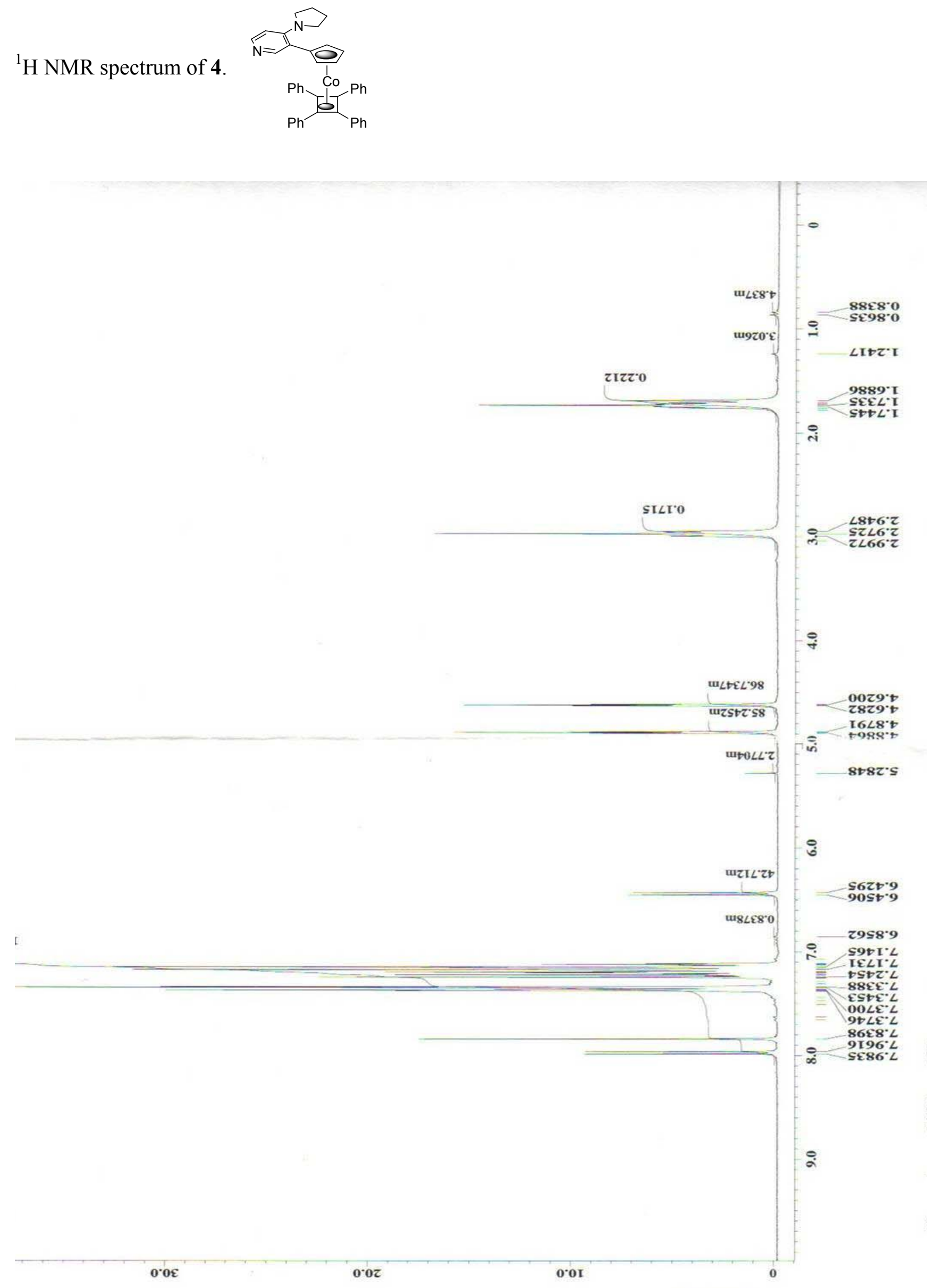

S8 
${ }^{1} \mathrm{H}$ NMR spectrum of 7 .
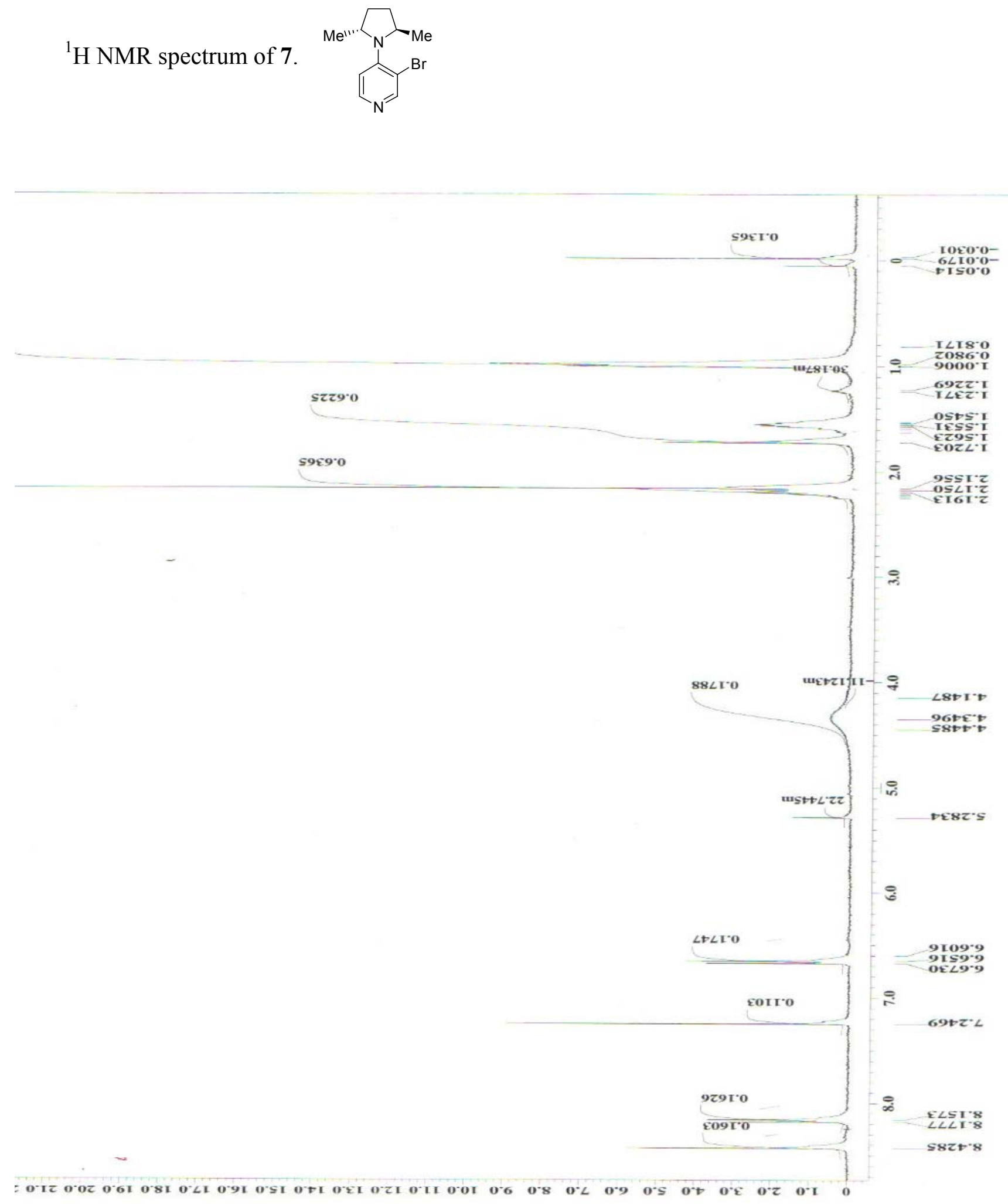
${ }^{1} \mathrm{H}$ NMR spectrum of $\mathbf{1}$.
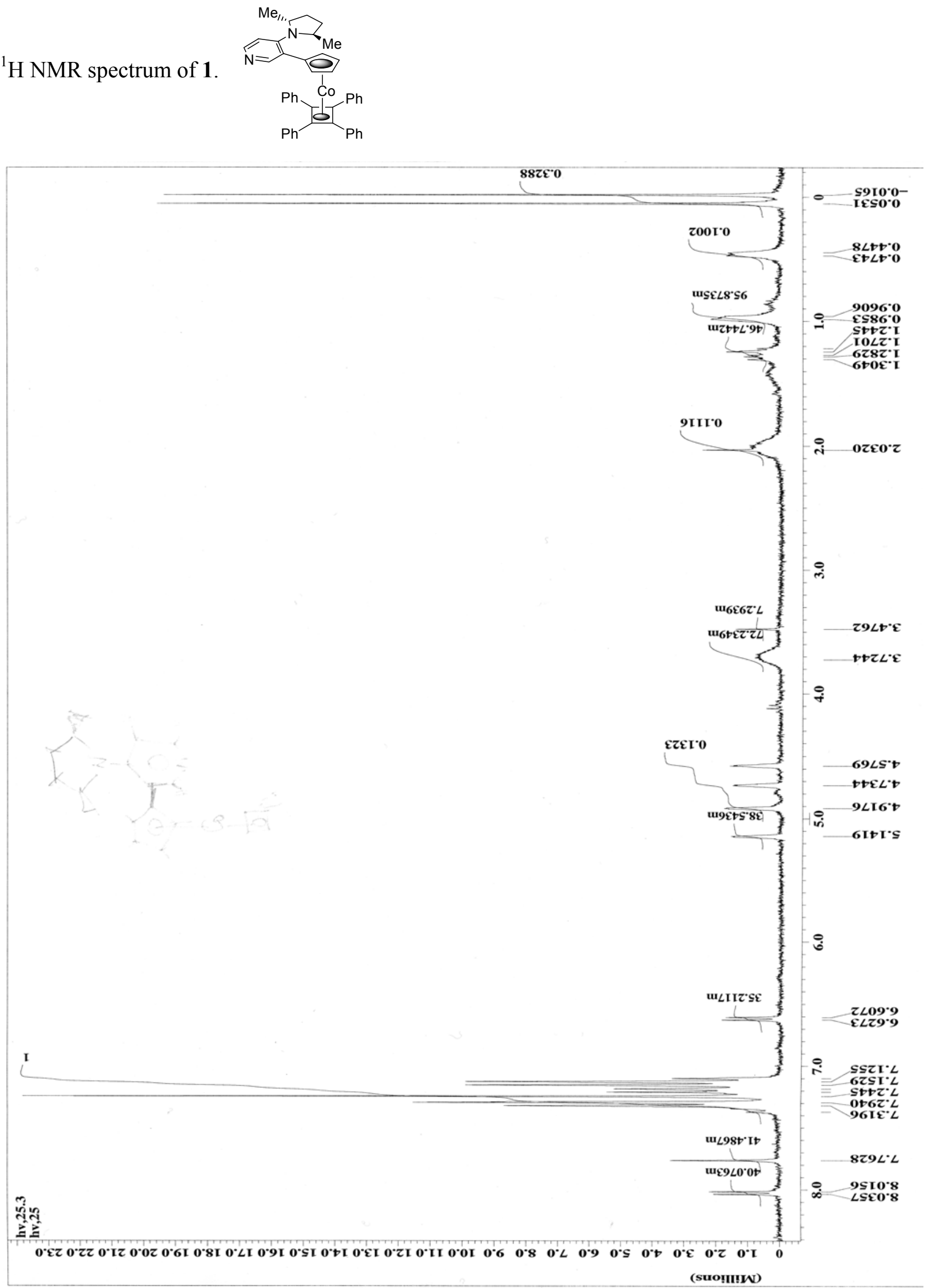
${ }^{1} \mathrm{H}$ NMR spectrum of
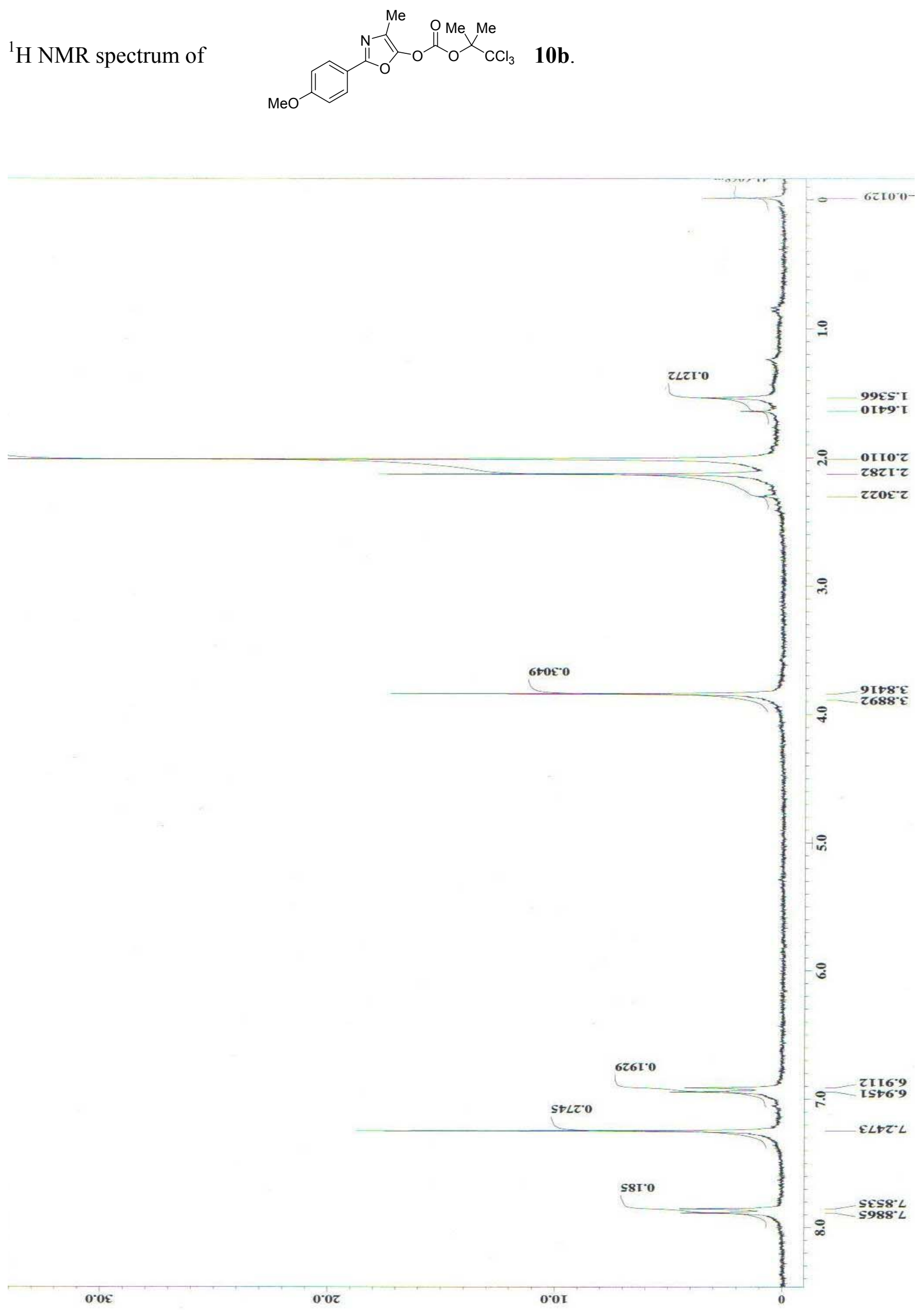

Si 

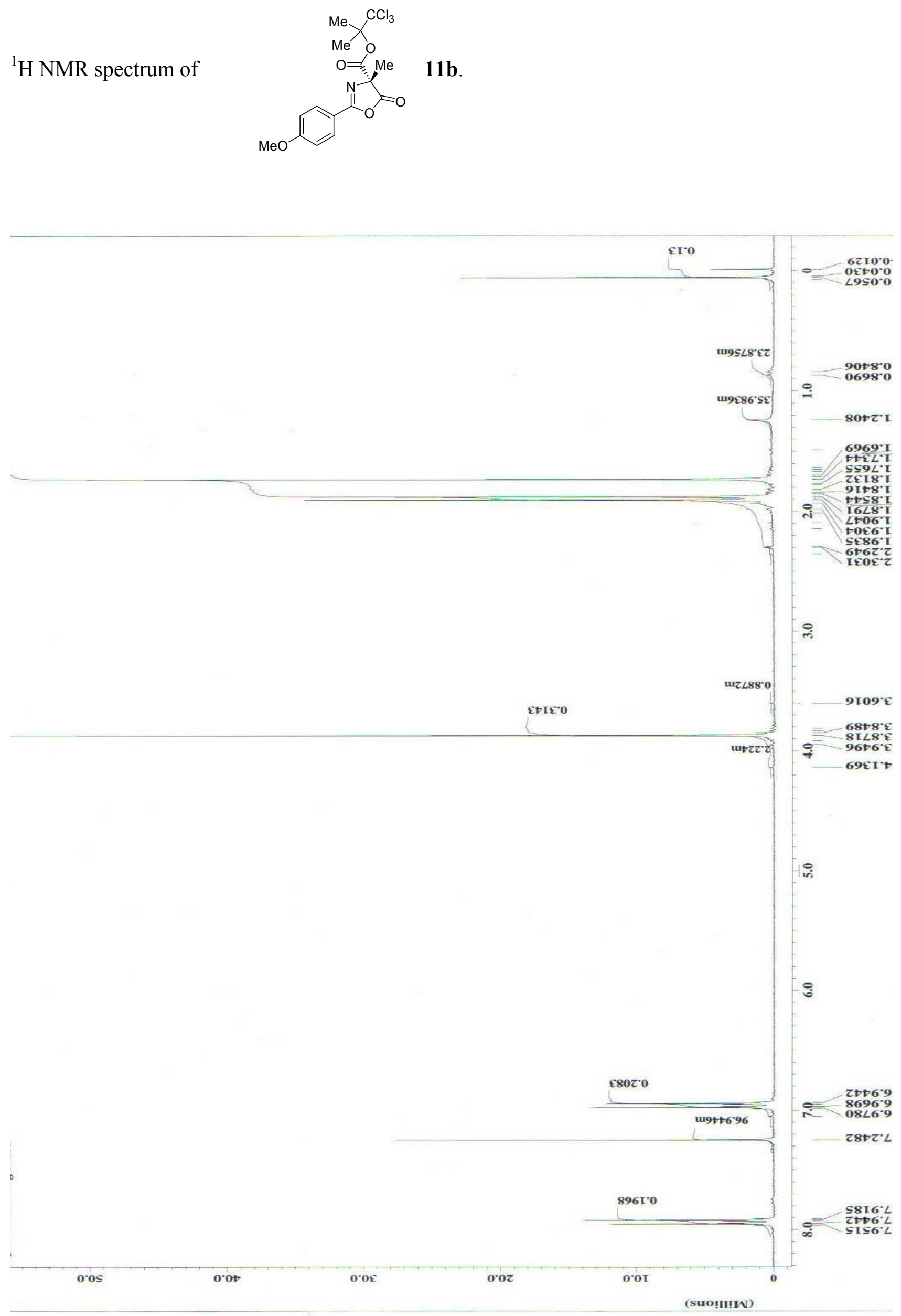
${ }^{1} \mathrm{H}$ NMR spectrum of

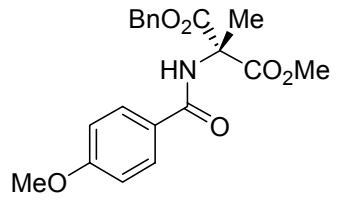

$12 \mathrm{a}$.

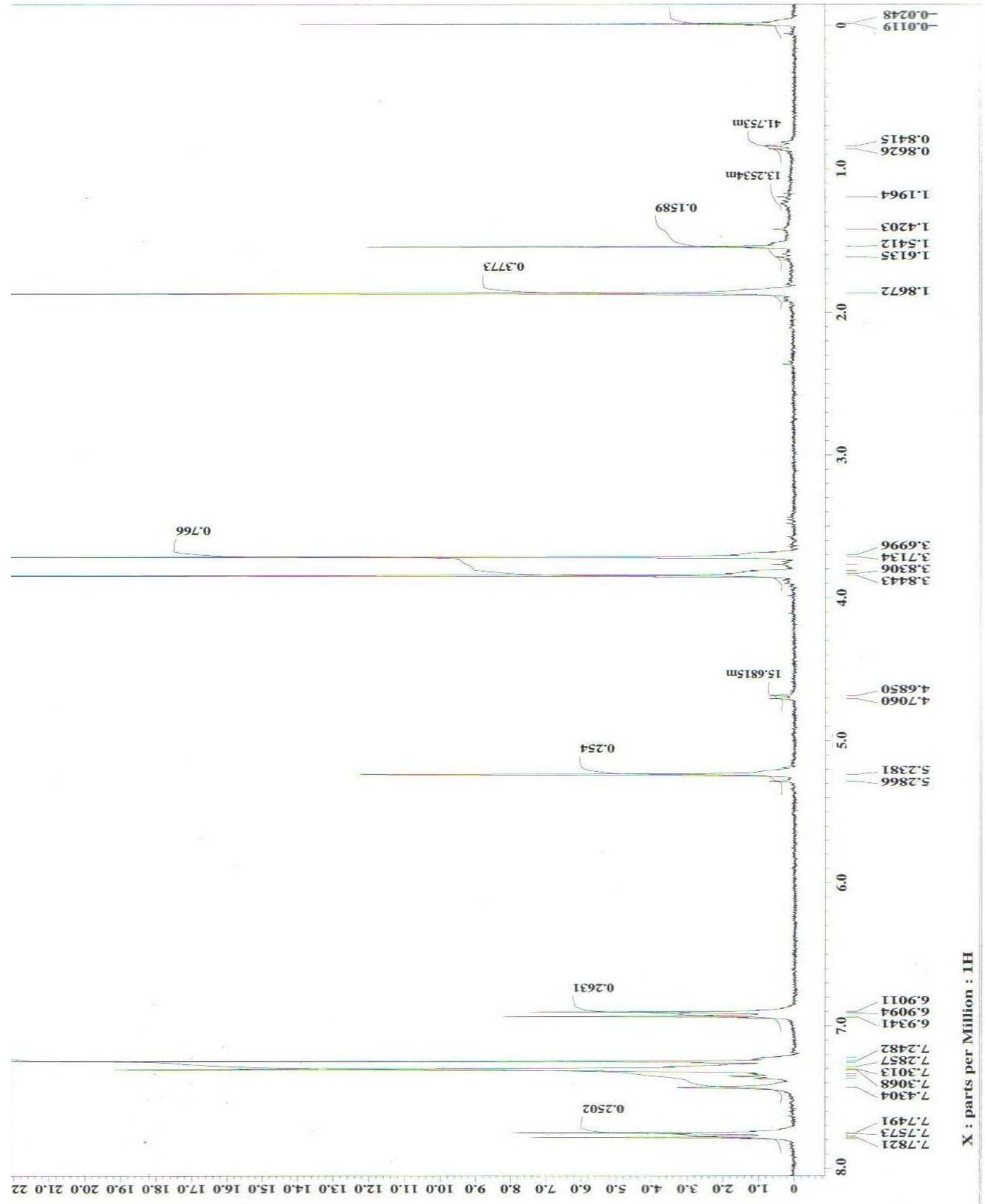


HPLC analysis of $\mathbf{1 1 b .}$<smiles>COc1ccc(C2=N[C@](C)(C(=O)OC(C)(C)C(C)(C)C)C(=O)O2)cc1</smiles>

HPLC (Chiralcel OD, 4.6mm $\times 25 \mathrm{~m}, 90: 10$ hexane/isopropanol, $1 \mathrm{~mL} / \mathrm{min}$ ) $\mathrm{T}_{\mathrm{R}}=13.25 \mathrm{~min}$ (major), $\mathrm{T}_{\mathrm{R}}=15.56 \mathrm{~min}$ (minor), $76 \%$ e.e.

Method Name: C:IVP_502EISElchiralcolumn method.met

Data Name: C:IVP 502E Instrument 2 Preview.dat

User: $\quad$ System

Acquired: $\quad$ 8/2/05 5:05:21 PM

Printed: $\quad$ 8/2/05 5:36:35 PM

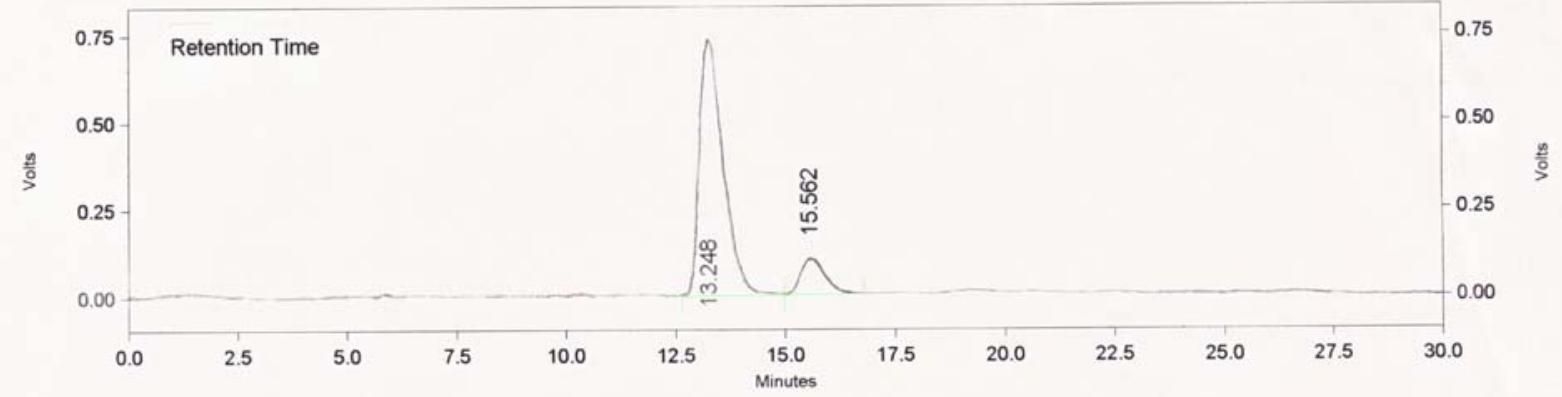

Channel A

\begin{tabular}{rrrrrr|}
\hline Time & Area & Area \% & Height & Height \% \\
\hline 13.248 & 27168503 & 87.78 & 736430 & 87.85 \\
15.562 & 3783409 & 12.22 & 101853 & 12.15 \\
\hline \multicolumn{2}{|c|}{ Totals } & 30951912 & 100.00 & 838283 & 100.00 \\
\hline
\end{tabular}


HPLC analysis of 12a.<smiles>COc1ccc(C(=O)NC(C)(C(C)=O)C(=O)O)cc1</smiles>

HPLC (Chiralcel OD, 4.6mm $\times 25 \mathrm{~m}, 90: 10$ hexane/isopropanol, $1 \mathrm{~mL} / \mathrm{min}$ ) $\mathrm{T}_{\mathrm{R}}=28.02 \mathrm{~min}$ (minor), $\mathrm{T}_{\mathrm{R}}=30.48 \mathrm{~min}$ (major), $75 \%$ e.e.

Method Name: C:IVP_502ElSElchiralcolumn method.met

Data Name: C:IVP 502EIInstrument 2 Preview.dat

User: $\quad$ System

Acquired: $\quad 6 / 21 / 05$ 11:11:20 AM

Printed: $\quad$ 6/21/05 11:58:25 AM

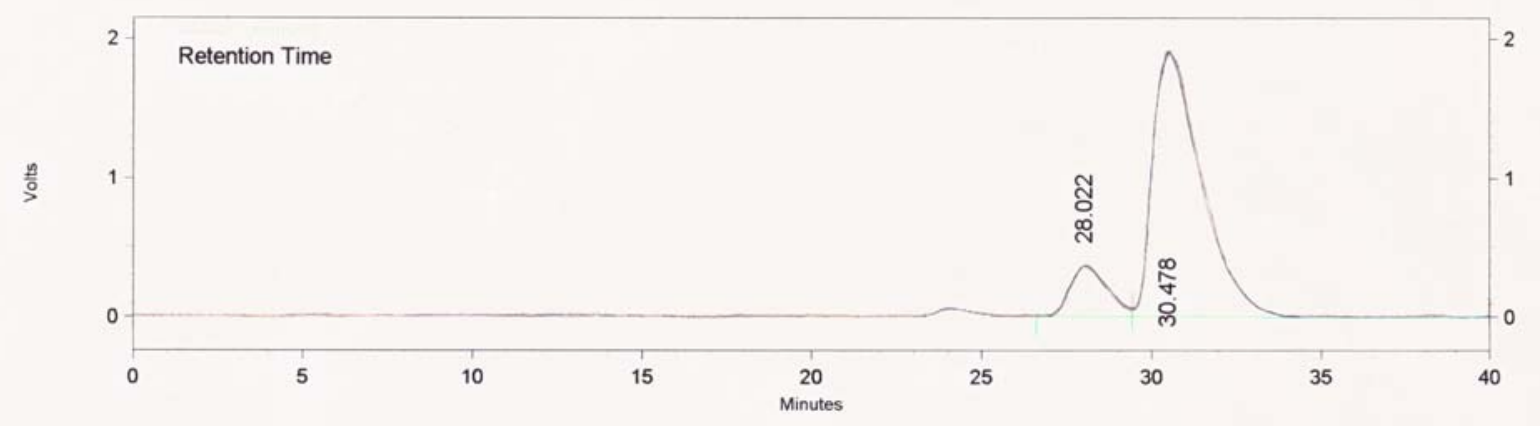

Channel A

\begin{tabular}{rrrrrr} 
Time & Area & Area \% & Height & Height \% \\
\hline \multicolumn{5}{c}{} & \\
28.022 & 26683596 & 12.32 & 358279 & 15.79 \\
30.478 & 189920395 & 87.68 & 1911370 & 84.21 \\
\hline Totals & 216603991 & 100.00 & 2269649 & 100.00 \\
\hline
\end{tabular}

